\title{
Corela
}

Cognition, représentation, langage

HS-1 1 | 2012

RJC Cotexte, contexte, situation

\section{L'accord sujet-verbe en français contemporain : une étude de la variation grammaticale et sociale}

\section{Anna Tristam}

\section{OpenEdition}

\section{Journals}

Édition électronique

URL : http://journals.openedition.org/corela/2614

DOI : $10.4000 /$ corela.2614

ISSN : $1638-573 \mathrm{X}$

\section{Éditeur}

Cercle linguistique du Centre et de I'Ouest - CerLICO

\section{Référence électronique}

Anna Tristam, «L'accord sujet-verbe en français contemporain : une étude de la variation grammaticale et sociale », Corela [En ligne], HS-11 | 2012, mis en ligne le 02 avril 2012, consulté le 19 avril 2019. URL : http://journals.openedition.org/corela/2614; DOI : 10.4000/corela.2614

Ce document a été généré automatiquement le 19 avril 2019

\section{(c) (i) (2)(2)}

Corela - cognition, représentation, langage est mis à disposition selon les termes de la licence Creative Commons Attribution - Pas d'Utilisation Commerciale - Partage dans les Mêmes Conditions 4.0 International. 


\title{
L'accord sujet-verbe en français contemporain : une étude de la variation grammaticale et sociale
}

\author{
Anna Tristam
}

\section{Introduction}

\subsection{L'accord sujet-verbe}

1 Lorsque l'on s'intéresse au vaste domaine de l'accord sujet-verbe, on sait que dans la plupart des cas le locuteur n'a pas le choix de l'accord - c'est-à-dire qu'il n'y a qu'un accord possible. Néanmoins, il existe des contextes dans lesquels on peut trouver une variation entre l'accord singulier et le pluriel (cf. Corbett 2006 ; Grevisse 1993 ; Riegel et al 1994). Cette variation est souvent liée à une discordance entre le nombre syntaxique et le nombre sémantique. C'est le cas de certains noms collectifs et de certaines expressions de quantité, comme dans les exemples suivants :

(1) Singulier :

" Une centaine de vétérans américains est également attendue »

(Ouest France, 23-24 mai 2009, p. 8)

«On a affaire à une minorité qui fait la loi à l'université »

(Ouest France, 23-24 mai 2009, p. 13)

(2) Pluriel :

«Un petit millier de producteurs allemands, français et belges se sont déplacés,

hier, à Bruxelles [...]»

(Ouest France, 26 mai 2009, p. 3)

" Une trentaine d'associations de malades [...] affirment que des médecins refusent des consultations à des patients pauvres "

(Ouest France, 26 mai 2009, p. 3)

La variation de l'accord entre le singulier et le pluriel se retrouve dans une gamme assez large d'expressions : par exemple, foule, multitude, tas, armée, nuée, série, majorité, minorité, 
moitié, partie, reste, tiers, dizaine, centaine, millier, etc. Bien que l'accord au singulier soit généralement perçu comme l'accord 'correct', la réalité nous montre que la situation est plus complexe: en guise de témoignage, on trouve dans des grammaires telles que Grevisse (1993) ou Riegel et al (1994) des sections entières consacrées à une seule expression et à la variation de l'accord sujet-verbe qu'elle induit. Ces expressions sont à la base de notre projet de recherche, qui les traitera à partir de deux perspectives différentes : une perspective sociolinguistique et une perspective linguistique.

\subsection{Perspectives linguistique et sociolinguistique : le cotexte et le contexte}

Il y a fort à penser (cf. Corbett 2006) que de nombreux facteurs internes, c'est-à-dire des facteurs linguistiques, jouent un rôle dans l'accord sujet-verbe de ces expressions: la distinction animé - non-animé, par exemple; la présence/absence d'un complément singulier/pluriel; le déterminant (défini/indéfini); ou bien l'expression elle-même (majorité, moitié, trentaine, etc.). Ici donc, dans une perspective linguistique, nous voyons l'influence du cotexte : les facteurs linguistiques qui jouent un rôle dans l'accord sujet-verbe concernant ces expressions.

Dans une perspective sociolinguistique, cette variation représente un domaine supplémentaire pour l'étude de la variation grammaticale en français, qui reste jusqu'à présent relativement mal connue, faute de données, notamment. La perspective sociolinguistique entre en jeu ici parce que l'accord au singulier peut être perçu comme le seul accord 'correct', si l'on s'en tient au nombre grammatical, et ce bien que les grammaires (cf. Grevisse 1993; Riegel et al 1994) fassent preuve d'une certaine confusion et d'hésitation sur l'accord 'correct', tout autant que les locuteurs eux-mêmes. L'accord que choisissent les locuteurs dans des contextes linguistiques et sociaux différents peut être lié à leurs caractéristiques sociales, mais aussi à leur sensibilité à des jugements normatifs, ainsi qu'au contexte particulier de l'interaction (formel, informel, spontané, non-spontané, oral, écrit...). C'est par ce biais que la variation de l'accord entre le singulier et le pluriel nous offre un aperçu de la variation sociale en français, et que le contexte (interprété ici dans le sens le plus large - les facteurs sociaux ainsi que la situation de l'interaction) ressort comme concept fondamental pour examiner l'accord sujet-verbe de ce type.

Dans ce qui suit, nous nous pencherons sur une première enquête de notre projet. Nous examinerons, en premier lieu, les buts et la méthodologie de cette première enquête, et nous regarderons, en second lieu, quelques résultats ayant traits aux facteurs sociaux, avant d'en tirer quelques conclusions pour notre étude principale.

\section{Une première enquête}

\subsection{Buts}

Cette première enquête avait pour but d'identifier les facteurs les plus importants pour l'étude principale - c'est-à-dire les aspects les plus pertinents du cotexte et du contexte ainsi que d'identifier les aspects problématiques de la méthodologie. Ici nous traiterons seulement des facteurs sociaux que nous avons pris en compte dans l'enquête : 
(i) l'âge du locuteur

(ii) le sexe du locuteur

(iii) le niveau d'éducation du locuteur

Dans ce qui suit, nous nous limitons donc à l'étude du contexte et de son influence sur l'accord sujet-verbe induit par ces expressions.

\subsection{Méthodologie}

8 Il faut noter que l'étude de la variation en syntaxe - par rapport à la variation en phonologie, par exemple - pose quelques difficultés théoriques (cf. Lavandera 1978, Sankoff 1980, Romaine 1981, Cheshire 1987; voir aussi Gadet 1997 pour un résumé), principalement parce ces variantes sont pertinentes pour la signification. Pour le dire brièvement, quand on a affaire à des variantes non pertinentes, ce qui est le cas des variantes phonologiques, on peut les considérer comme équivalentes dans tous les contextes dans lesquels elles se trouvent. En revanche, au-delà de la phonologie - dans le domaine de la morphosyntaxe par exemple - les variantes sont nécessairement pertinentes. Il faut donc aborder la question de l'équivalence sémantique : peut-on dire que ces variantes (pour nous, le singulier et le pluriel) sont des équivalents sémantiques dans tous les contextes où elles se trouvent? Pour prendre un exemple (singulier dans la version originale) :

(3) Singulier :

"On a affaire à une minorité qui fait la loi à l'université "

(Ouest France, 23-24 mai 2009, p. 13)

(4) Pluriel :

« On a affaire à une minorité qui font la loi à l'université »

Il est vraisemblable qu'il y ait ici une légère différence d'interprétation selon l'accord: le singulier met l'accent sur l'unanimité (réelle ou imaginée) du groupe ; le pluriel change la focalisation de la phrase, en la transférant sur les individus qui forment ce groupe, au lieu du groupe comme unité. Il ne fait pas de doute que des facteurs sémantiques et pragmatiques vont jouer un rôle important dans l'accord avec les expressions de quantité que nous examinons ici. Toutefois, étant donné que nous nous intéressons plutôt dans cette communication aux facteurs sociaux qui peuvent avoir une influence sur l'accord sujet-verbe induit par ces expressions de quantité, nous supposerons pour l'instant une équivalence sémantique provisoire qui suffira pour la première enquête dont il s'agit ici.

Il est un autre problème, de nature plutôt méthodologique cette fois-ci, qui entre en jeu quand on a affaire à des variantes morphosyntaxiques. L'occurrence des variantes dans la conversation dépend largement des sujets qui y sont traités, et par conséquent il n'est pas toujours facile d'obtenir des données réelles pour en faire une analyse. Dans ce projet de recherches, nous utilisons deux techniques principales pour éviter ce problème :

(i) des entretiens sociolinguistiques

(ii) des exercices écrits (des exercices à trous)

Les entretiens sociolinguistiques ont pour but d'encourager l'utilisation des expressions ciblées dans cette étude, et d'évoquer des exemples de ces expressions dans une conversation assez informelle. Dans l'exercice écrit, en revanche, les participants doivent compléter une phrase qui contient une des expressions auxquelles nous nous intéressons, dans des conditions où nous pouvons contrôler plus précisément les facteurs linguistiques qui entrent en jeu. L'utilisation de ces deux techniques nous offre une perspective supplémentaire, qui est la comparaison entre l'usage spontané et l'usage non- 
spontané. Cette comparaison nous permet d'observer à quel point le contexte («situationnel») influence l'usage linguistique des locuteurs. L'usage des locuteurs change-t-il selon le contexte? Montrent-ils plus de sensibilité aux normes dans leur usage non-spontané, par exemple?

\subsection{Résultats de l'exercice écrit (âge, sexe, éducation)}

Les résultats présentés ici ne concernent que l'exercice écrit pour les facteurs sociaux que nous avons mentionnés plus haut (âge, sexe, éducation). L'exercice écrit compte 40 questions, dont 15 qui contiennent une des expressions de quantité ciblées, et 25 qui permettent de dissimuler l'objectif de l'exercice. L'ordre des questions est aléatoire. Dans chaque cas, le participant doit conjuguer au passé composé le verbe donné entre parenthèses (toujours avec l'auxiliaire avoir), par exemple :

(5)

Un magazine masculin révèle que la majorité des hommes (avoir) des problèmes de sommeil.

Selon le sondage, une minorité de députés (travailler) dans

d'autres domaines avant de faire de la politique.

Dans la confusion, une foule de personnes _-_-_-_-_-_-_-_-_-_ (pouvoir) s'échapper.

13 Pour calculer les résultats de chaque participant, le nombre de réponses au singulier et au pluriel est noté. Il y a aussi d'autres réponses dont il faut tenir compte : soit le participant, au lieu de conjuguer le verbe au passé composé, a utilisé un temps dans lequel le singulier et le pluriel sont homophones (e.g. l'imparfait) ; soit le participant a donné le singulier ET le pluriel ; soit la réponse donnée était illisible; etc. Ces réponses ont été notées comme 'Autre'.

\subsection{1 Âge}

Il faut avouer que notre corpus est un peu déséquilibré au niveau de la répartition des participants par âge : la première enquête a été effectuée à Cambridge, au Royaume Uni, et il n'a pas toujours été possible de trouver un nombre égal de participants dans chaque catégorie. 


\begin{tabular}{|c|c|c|c|c|c|c|}
\hline Participant & Age & Sexe & Éducation & SING & PLU & AUTRE \\
\hline $119-\mathrm{C} / \mathrm{LK}-\mathrm{T}$ & 16 & F & $-B A C$ & 1 & 6 & 8 \\
\hline $219-\mathrm{L} / \mathrm{CK}-\mathrm{T}$ & 18 & $\mathrm{~F}$ & $-B A C$ & 4 & 11 & 0 \\
\hline $318-\mathrm{TB}-\mathrm{T}$ & 19 & $\mathrm{H}$ & $-B A C$ & 5 & 10 & 0 \\
\hline 405 SCLS-T & 21 & F & + UNIV & 8 & 6 & 1 \\
\hline $513 \mathrm{FP}-\mathrm{V}$ & 21 & F & + UNIV & 14 & 1 & 0 \\
\hline $606 \mathrm{NLM}-\mathrm{T}$ & 22 & F & +UNIV & 12 & 3 & 0 \\
\hline $812 \mathrm{CR}-\mathrm{V}$ & 22 & $\mathrm{H}$ & $+\mathrm{BAC}$ & 5 & 8 & 2 \\
\hline $711 \mathrm{FD}-\mathrm{T}$ & 22 & $\mathrm{H}$ & + UNIV & 5 & 10 & 0 \\
\hline 9 LBC & 23 & F & + UNIV & 9 & 5 & 1 \\
\hline $1010 \mathrm{DM}-\mathrm{V} / \mathrm{T}$ & 29 & $\mathrm{H}$ & + UNIV & 15 & 0 & 0 \\
\hline 1107 CG-T & 30 & $\mathrm{H}$ & + UNIV & 11 & 4 & 0 \\
\hline $12 \mathrm{DM}$ & 32 & $\mathrm{H}$ & +UNIV & 15 & 0 & 0 \\
\hline 1309 ST-V & 35 & F & + UNIV & 12 & 3 & 0 \\
\hline 14 04MS-T & 36 & F & + UNIV & 14 & 1 & 0 \\
\hline 15 08 JNC-T & 37 & $\mathrm{H}$ & + UNIV & 12 & 3 & 0 \\
\hline $1616 \mathrm{AO}-\mathrm{V}$ & 57 & F & + UNIV & 3 & 12 & 0 \\
\hline $1717 \mathrm{AK}-\mathrm{V}$ & 63 & F & + UNIV & 8 & 1 & 6 \\
\hline
\end{tabular}

Dans le tableau 1 les résultats pour chaque individu sont présentés, divisés en quatre tranches d'âge : moins de 19 ans (les participants 1-3), 20-29 ans (les participants 4-10), 30-39 ans (les participants 11-15) et 50 ans et plus (les participants 16 et 17). Le nombre de réponses au singulier et au pluriel y est donné, ainsi que les réponses traitées comme 'Autres'. On peut voir que les participants dans la tranche d'âge 30-39 ans ont tendance à répondre plus souvent au singulier que les participants des autres groupes.

Tableau 2 : Moyenne des résultats - âge

\begin{tabular}{lccc}
\multicolumn{5}{c}{ Moyenne } \\
Age & SING & PLU & AUTRE \\
$<19$ & 3.33 & 9.00 & 2.67 \\
$20-29$ & 9.71 & 4.71 & 0.57 \\
$30-39$ & 12.80 & 2.20 & 0.00 \\
$>50$ & 5.50 & 6.50 & 3.00
\end{tabular}

16 Si on regarde la moyenne des résultats dans le tableau 2, on peut voir plus clairement en quoi les tranches d'âge diffèrent: les participants les plus jeunes $(<19)$ ont répondu plus souvent par le pluriel que par le singulier (9/14 en moyenne pour le pluriel, contre 3.33/14 pour le singulier). Dans la tranche des 20 à 29 ans, c'est plutôt l'inverse : ces participants ont utilisé en moyenne plus de réponses au singulier qu'au pluriel (9.71/14 contre 4.71).On observe le même phénomène chez les 30-39 ans, où la différence est 
encore plus marquée. Pour les participants de 50 ans et plus, étant donné qu'il n'y a que deux participants, et que la moyenne cache des réponses individuelles assez différentes (voir Tableau 1), on ne peut pas tirer de conclusions certaines. Globalement, le test du chi carré confirme que l'âge du locuteur est un facteur très important $(\mathrm{p}=<0.0001)$.

Avant de nous pencher sur les résultats concernant le sexe et le niveau d'éducation des participants, notons que l'âge du locuteur entrera en jeu avec d'autres facteurs, comme par exemple le niveau d'éducation - les participants les plus jeunes, par exemple, ne passent leur bac qu'à l'âge de 18 ans, en moyenne.

\subsubsection{Sexe}

L'échantillon de participants par sexe est assez bien équilibré, sauf pour les participants de 50 ans et plus: dans cette catégorie, il n'y a que des femmes. Si l'on regarde les résultats par individu et en moyenne (tableaux 3 et 4, ci-dessous), on observe peu de différence entre le comportement des hommes et celui des femmes au niveau de leurs réponses (même s'il y a des comportements différents à l'intérieur de chaque catégorie, qui tiennent à d'autres facteurs). Le test du chi carré confirme que le sexe est statistiquement significatif, mais seulement au niveau de $0.05(p=0.025)$.

Tableau 3 : Résultats par individu - sexe

\begin{tabular}{lcccccc} 
Participant & Sexe & Age & Éducation & SING & PLU AUTRE \\
1 19-C/LK-T & F & 16 & - BAC & 1 & 6 & 8 \\
$219-$ LCK-T & F & 18 & - BAC & 4 & 11 & 0 \\
3 05SCLS-T & F & 21 & + UNIV & 8 & 6 & 1 \\
413 FP-V & F & 21 & + UNIV & 14 & 1 & 0 \\
5 06NLM-T & F & 22 & + UNIV & 12 & 3 & 0 \\
6 LBC & F & 23 & + UNIV & 9 & 5 & 1 \\
7 09ST-V & F & 35 & + UNIV & 12 & 3 & 0 \\
804 MS-T & F & 36 & + UNIV & 14 & 1 & 0 \\
916 AO-V & F & 57 & + UNIV & 3 & 12 & 0 \\
1017 AK-V & F & 63 & + UNIV & 8 & 1 & 6 \\
\hline 11 18-TB-T & H & 19 & - BAC & 5 & 10 & 0 \\
1312 CR-V & H & 22 & + BAC & 5 & 8 & 2 \\
1211 FD-T & H & 22 & + UNIV & 5 & 10 & 0 \\
1410 DM-V T & H & 29 & + UNIV & 15 & 0 & 0 \\
15 07CG-T & H & 30 & + UNIV & 11 & 4 & 0 \\
16 DM & H & 32 & + UNIV & 15 & 0 & 0 \\
17 08JNC-T & H & 37 & + UNIV & 12 & 3 & 0
\end{tabular}


Tableau 4 : Moyenne des résultats - sexe

\begin{tabular}{lccc}
\multicolumn{3}{c}{ Moyenne } \\
Sexe & SING & PLU & AUTRE \\
Femmes & 8.50 & 4.90 & 1.60 \\
Hommes & 9.71 & 5.00 & 0.29
\end{tabular}

Bien que ce résultat puisse paraître surprenant quand on le considère à la lumière des études classiques en sociolinguistique (Labov 1972, Trudgill 1972, etc. ; voir Cheshire 2002 pour sommaire), qui témoignent en général d'un effet fort marqué du sexe du locuteur sur sa façon de parler, les études sociolinguistiques portant sur le français ont montré que la relation entre le sexe et d'autres facteurs sociaux comme le métier ou la mobilité sociale est peut-être plus complexe qu'on n'aurait pu le penser (cf. Armstrong et al 2001).

\subsection{3 Éducation}

Pour ce qui est de l'éducation comme facteur social, dans cette enquête préliminaire nous cherchons des participants de trois niveaux d'éducation différents : des participants sans le baccalauréat (-BAC), des participants avec le baccalauréat mais sans études supérieures $(+B A C)$, et des participants diplômés d'études supérieures (+UNIV). Malheureusement, dans cette première enquête, la répartition des participants par niveau d'éducation n'a pas été idéale : il n'y a eu que 3 participants sans le bac (participants 1-3), et un seul participant avec le bac mais sans études supérieures (participant 4); en revanche, dans la catégorie +UNIV il y a eu 13 participants (5-17).

Tableau 5 : Résultats par individu - éducation

\begin{tabular}{|c|c|c|c|c|c|c|}
\hline Participant & Éducation & Âge & Sexe & SING & PLU & AUTRE \\
\hline $119-\mathrm{C} / \mathrm{LK}-\mathrm{T}$ & - BAC & 16 & F & 1 & 6 & 8 \\
\hline 2 19-L/CK-T & $-B A C$ & 18 & $\mathrm{~F}$ & 4 & 11 & 0 \\
\hline 3 18-TB-T & $-B A C$ & 19 & $\mathrm{M}$ & 5 & 10 & 0 \\
\hline $412 \mathrm{CR}-\mathrm{V}$ & $+\mathrm{BAC}$ & 22 & $\mathrm{M}$ & 5 & 8 & 2 \\
\hline 505 SCLS-T & + UNIV & 21 & $\mathrm{~F}$ & 8 & $\overline{6}$ & 1 \\
\hline $613 \mathrm{FP}-\mathrm{V}$ & + UNIV & 21 & $\mathrm{~F}$ & 14 & 1 & 0 \\
\hline $706 \mathrm{NLM}-\mathrm{T}$ & + UNIV & 22 & $\mathrm{~F}$ & 12 & 3 & 0 \\
\hline \& 11FD-T & + UNIV & 22 & M & 5 & 10 & 0 \\
\hline $9 \mathrm{LBC}$ & + UNIV & 23 & $\mathrm{~F}$ & 9 & 5 & 1 \\
\hline 10 10DM-V/T & + UNIV & 29 & M & 15 & 0 & 0 \\
\hline 11 07CG-T & + UNIV & 30 & M & 11 & 4 & 0 \\
\hline $12 \mathrm{DM}$ & + UNIV & 32 & M & 15 & 0 & 0 \\
\hline 13 09ST-V & + UNIV & 35 & $\mathrm{~F}$ & 12 & 3 & 0 \\
\hline 14 04MS-T & + UNTV & 36 & $\mathrm{~F}$ & 14 & 1 & 0 \\
\hline 15 08JNC-T & + UNIV & 37 & M & 12 & 3 & 0 \\
\hline $1616 \mathrm{AO}-\mathrm{V}$ & + UNIV & 57 & $\mathrm{~F}$ & 3 & 12 & 0 \\
\hline $1717 \mathrm{AK}-\mathrm{V}$ & + UNIV & 63 & $\mathrm{~F}$ & 8 & 1 & 6 \\
\hline
\end{tabular}


21 Malgré ces difficultés, les résultats témoignent d'une différence de comportement entre des participants au niveau d'éducation différents : si l'on regarde le tableau des résultats par individu (tableau 5), on observe que les participants sans études supérieures (-BAC et $+\mathrm{BAC})$ ont tendance à mettre plus de réponses au pluriel que les participants diplômés d'études supérieures (+UNIV). Le test du chi-deux montre que, globalement, ces résultats sont statistiquement significatifs $(\mathrm{p}=<0.001)$. Le tableau 6 montre la moyenne des résultats pour l'éducation pour faciliter une comparaison avec les tableaux 2 et $4:$ notre catégorie $+B A C$ ne contient qu'un seul participant donc ici la moyenne est similaire aux résultats bruts.

Tableau 6 : Moyenne des résultats - éducation

\begin{tabular}{|c|c|c|c|}
\hline \multicolumn{3}{|c|}{ Moyenne } & \\
\hline Éducation & SING & PLU & AUTRE \\
\hline$-\mathrm{BAC}$ & 3.33 & 9.00 & 2.67 \\
\hline$+\mathrm{BAC}$ & 5.00 & 8.00 & 2.00 \\
\hline + UNIV & 10.62 & 3.77 & 0.62 \\
\hline
\end{tabular}

\subsection{Autres remarques}

Les tableaux précédents ont montré que l'âge et l'éducation semblent être des facteurs importants dans la variation entre l'accord singulier et le pluriel avec les expressions qui nous intéressent. Pour ces variantes, cependant, le sexe ne semble pas être aussi significatif que les autres facteurs, du moins selon les résultats de cette première enquête.

3 Qui plus est, dans notre première enquête, nous avons vu quelques effets plutôt inattendus, qui sont particulièrement intéressants, étant donné que nous nous intéressons à l'influence du contexte (facteurs sociaux, normes et prescriptions, contexte proprement dit) sur l'usage réel des locuteurs de langue française. À cet égard, il est vraiment intéressant de constater que plusieurs participants ont donné les deux réponses possibles - c'est-à-dire qu'ils ont voulu montrer qu'ils accepteraient les deux accords, ou bien qu'ils n'ont pas voulu choisir entre les deux. Plus intéressant encore, certains participants ont écrit leur réponse de sorte qu'il nous est impossible de voir s'ils avaient choisi le singulier ou le pluriel. En outre, plusieurs participants ont choisi de mettre tous les accords au singulier dans l'exercice écrit, alors même qu'ils n'avaient utilisé que l'accord au pluriel pendant l'entretien.

Ce comportement témoigne peut-être d'une certaine hypercorrection chez quelques locuteurs. Ils sont conscients du fait que l'exercice écrit est une sorte de 'test'; ils sont aussi conscients du fait qu'il existe des normes et des règles pour ces expressions. Cependant, ils ne sont pas tout à fait sûrs de ce que sont ces normes, et le résultat est qu'ils se comportent d'une manière qui ne représente ni leur comportement naturel et spontané, ni un comportement qui suit les «règles». C'est peut-être aussi l'expression d'une sorte d'insécurité linguistique dans certains contextes qui semble exister chez les Français, ce qui n'a rien de surprenant, étant donné que la langue française est une des langues les plus codifiées au monde. 


\section{Quelques conclusions}

Notre première enquête nous a montré que le contexte - dans son sens le plus large - est très important pour l'accord sujet-verbe avec les expressions de quantité auxquelles nous nous intéressons ici : l'âge et le niveau d'éducation du locuteur ressortent comme des facteurs sociaux importants pour notre étude, tandis que le sexe semble être moins important. Néanmoins, dans l'étude principale, pour assurer une répartition équilibrée des participants, nous allons sélectionner un nombre égal de participants hommes et femmes. Le contexte dans son sens le plus restreint est aussi apparu comme un facteur important dans notre étude: l'usage de certains participants varie beaucoup selon le contexte spontané, informel ou non-spontané, formel, et ils se montrent sensibles aux normes et prescriptions linguistiques dans l'exercice écrit. La combinaison des deux techniques que nous avons choisies semble convenir aux objectifs de notre étude.

\section{BIBLIOGRAPHIE}

Armstrong, N. Bauvois, C., Beeching, K. avec Bruyninckx, M. 2001. La Langue française au féminin. (Paris : L'Harmattan)

Cheshire, J. 1987. 'Syntactic Variation, the Linguistic Variable and Sociolinguistic Theory', Linguistics $25: 257-282$

Cheshire, J. 2002. 'Sex and gender in variationist research', in J.K.Chambers, P. Trudgill and N. Schilling-Estes (eds.), Handbook of Language Variation and Change. (Oxford: Blackwell) (pp. 423-43)

Corbett, G. 2006. Agreement. (Cambridge: Cambridge University Press)

Gadet, F. 1997. 'La variation, plus qu'une écume', Langue Française 115 : 5-18

Grevisse, M. 1993. Le bon usage : Grammaire française. 13e édition refondue par André Goosse. (Paris: Duculot)

Labov, W. 1972. Sociolinguistic Patterns. (Philadelphia: University of Pennsylvania Press)

Lavandera, B.R. 1978. 'Where Does the Sociolinguistic Variable Stop?', Language in Society 7: 171-82

Riegel, M., J-C. Pellat \& R. Rioul. 1994. Grammaire méthodique du français. (Paris: Presses Universitaires de France)

Romaine, S. 1981. 'On the Problem of Syntactic Variation: a Reply to Beatriz Lavandera and William Labov', Working Papers in Sociolinguistics 82: 1-38

Sankoff, G. 1980. 'Above and Beyond Phonology in Variable Rules', in Sankoff, G. (ed.) The Social Life of Language. (Philadelphia: University of Pennsylvania Press)

Trudgill, P. 1972. 'Sex, covert prestige and linguistic change in the urban British English of Norwich', Language in Society 1: 179-95 


\section{RÉSUMÉS}

Dans cet article, nous évaluons le rôle du cotexte (les facteurs internes ou linguistiques) et du contexte (les facteurs externes ou sociaux, et la situation de l'interaction) dans l'accord sujetverbe induit par les expressions de quantité en français contemporain. Dans l'emploi de ces expressions, par exemple la majorité, le reste etc., il existe une variation entre l'accord singulier et le pluriel, qui est liée à une discordance entre le nombre syntaxique et le nombre sémantique de l'expression. Dans un premier temps, nous présentons la méthodologie d'une première enquête dont le but était d'identifier les principaux facteurs internes et externes qui ont une influence sur les accords de ce type. Dans un deuxième temps, nous présentons les résultats pour les facteurs sociaux avant d'en tirer quelques conclusions pour l'étude principale.

In this paper, the results from a pilot study of a larger project looking at variable agreement with quantifying expressions in French are reported. Quantifying expressions such as 'la majorité, 'le reste' can be found with either singular or plural agreement in French, which is often attributed to a mismatch between the syntactic and semantic number of the noun. This project considers these expressions from both a linguistic and a sociolinguistic perspective, looking at the effect of a number of linguistic and social factors on agreement, and considering therefore the influence of the cotext (internal factors) and context (external factors). The aim of the pilot study reported here was to identify the most important factors for consideration in the main study, and to identify any possible problems with the methodology.

\section{INDEX}

Mots-clés : variation grammaticale, variation sociolinguistique, accord sujet-verbe

Keywords : grammatical variation, sociolinguistic variation, subject-verb agreement

\section{AUTEUR}

ANNA TRISTAM

Université de Cambridge / Clare College, Royaume Uni 